\title{
Developmental Assessment in Low Birth Weight Infants at One Year of Age using Ages and Stages Questionnaire
}

\author{
Ali Dehghani ${ }^{1}(\mathbb{D})$, Marzieh Sobhani ${ }^{* 1(\mathbb{D}}$, Mahmoud Nouri Shadkam ${ }^{2}{ }^{\mathbb{D}}$, Hossein Falahzadeh ${ }^{1}$, \\ Masoud Mohammadi ${ }^{3}$ (D), Aboalfazl Sharifi 4 (D) \\ 1. Dept. of Statistics and Epidemiology, School of Health, Shahid Sadoughi University of Medical Sciences and Health \\ Services, Yazd, Iran \\ 2. Maternal and Neonatal Health Research Center, Shahid Sadoughi University of Medical Sciences and Health Services, \\ Yazd, Iran \\ 3. Dept. of Nursing, School of Nursing and Midwifery, Kermanshah University of Medical Sciences, Kermanshah, Iran \\ 4. Dept. of Statistics and Epidemiology, School of Health, Zahedan University of Medical Sciences, Zahedan, Iran
}

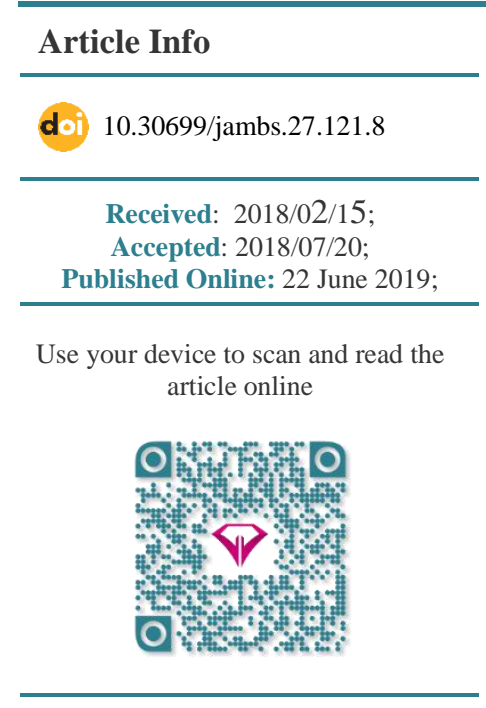

Corresponding Information: Marzieh Sobhani, Dept. of Statistics and Epidemiology, School of Health, Shahid Sadoughi University of Medical Sciences and Health Services, Yazd, Iran Email: sobhani9469@gmail.com

\begin{abstract}
Background \& Objective: Low birth weight is considered as one the causes of infant mortality across the world. Accordingly, paying attention to neonatal growth leads to mortality rate decrement and consequently prevents future physical and mental disabilities in this age group.
\end{abstract}

Materials \& Methods: This historic (retrospective) cohort study was conducted on 280 one-year-old male and female infants who were divided into two equal groups of low and normal birth weights. Then, the developmental status of the neonates at the corrected age of one year was assessed using the Ages and Stages Questionnaire (ASQ). Data analysis was performed using SPSS 19 via $t$-test and multiple regression analysis.

Results: Of the 280 infants examined, $142(50.7 \%)$ of them were male and 138 $(49.3 \%)$ were female with the mean weight of $640.9 \pm 2696.9 \mathrm{gr}$, and the mean gestational age of $37.8 \pm 2.4$ weeks. Moreover, the findings revealed that the gross motor skills and the problem-solving areas had the highest levels of developmental delay in the subjects. In addition, a significant relationship was observed between the developmental delay of the neonates in different areas and the variables of birth weight, birth height, birth rate, duration of hospitalization in neonatal intensive care units (NICUs), and some causes of neonatal hospitalization $(P<0.05)$.

Conclusion: According to the results, health policymakers were recommended to pay much more attention to the assessment of the developmental status of low-weight neonates for timely diagnosis and treatment as well as the education of health care providers and parents.

Keywords: Developmental status, Low birth weight, Ages and Stages Questionnaire (ASQ)

\section{Introduction}

Low birth weight (LBW) is one of the common healthcare problems and an important cause of neonatal mortality in the world (1). Infants with LBW are categorized into groups of moderately low birth weight (MLBW) with the weight of 1500-2499 gr, very low birth weight (VLBW) with the weight of below $1500 \mathrm{gr}$, and extremely low birth weight (ELBW) with the weight of below $1000 \mathrm{gr}$ (2). According to the statistics reported by the World Health Organization (WHO), there is a $17 \%$ rate of $\mathrm{LBW}$ in the world $(6 \%$ in industrial and $21 \%$ in developing countries), $10 \%$ rate in Iran and $8.4 \%$ in Yazd (3). Meanwhile, the highest neonatal mortality rate is allocated to the neonate age group in Iran (4), 50\% of which is related to LBW (5).
In general, LBW has a negative impact on neonatal evolution, quality of life, and financial burden on the healthcare system (2). In the recent decades, the advancement of science has increased the possibility of the survival of infants with LBW. Nevertheless, this escalation of survival has led to the increased complications caused by LBW, including increased population of children with cerebral palsy, seizure, hydrocephalus, blindness, deafness and cognitive disorders (5). In addition, LBW is associated with major damages in motor behaviors, which remain in childhood (1). According to the literature, the first two years are the most important periods of life since they are regarded as the most significant period for the brain development of infants with an important role in the mental, physical, social, and psychological performance of children (6). 
"Growth in the form of increased body size and development refers to the improved function of processes related to the body and mind" (7). In other words, lack of appearance of developmental properties specific to the age of infants is defined as developmental delay (8). In general, there are three types of developmental delay; including motor impairment (cerebral palsy), as well as behavioral and cognitive disorders (9). The recent studies have shown that infants with LBW experience a higher rate of neurological problems and developmental delay, compared to the other neonates (1). Moreover, lowweight infants are more prone to developmental delay in their gross and fine motor skill (10). Meanwhile, about $16 \%$ of children have developmental delay (3). Therefore, one of the priorities of the healthcare systems of developed countries is the developmental delay with the prevalence rate of $15-20 \%$ (7).

The American Academy of Pediatrics has recommended the implementation of early screening programs at various neonatal ages (e.g., 9, 18, 24 and 30 months) by healthcare providers for early diagnosis of developmental delay in infants with or without LBW (3). The most valuable measure taken for the early diagnosis of mental, motor, vision, and hearing impairments is the evaluation of the neonatal development. Treatment of developmental delay is possible if intervention is carried out in a timely manner. Otherwise, intervention could lead to permanent adverse outcomes (9). One of the major neural growth disabilities in these infants is cerebral palsy (10)

It is noteworthy that while the prevalence of cerebral palsy caused by infection during pregnancy has significantly decreased, LBW and premature birth have increased its prevalence rate again, leading to a new generation of cerebral palsy, especially in the developed countries (1). Assessment of neonatal development during the first year of life can reveal many defects, which can be treated with interventions and will eventually demonstrate the next neonatal state. Therefore, evaluation of the developmental status of these infants is crucial for the treatment and informing of parents. Given the difficulties in the screening of all neonates, conducting studies on high-risk newborns seems more logical. With regard to the high prevalence of LBW in neonates of Yazd, Iran and importance of the subject and unfavorable outcomes associated with LBW, the study aimed to evaluate the developmental status of infants with LBW at the corrected age of one year.

\section{Materials and Methods}

In this historical cohort study (the ethics code: IR.SSU.SPH.REC.1395.157), two health service centers were randomly selected from each geographic area of north, south, east, west, and center of Yazd. From each selected center, 140 infants with LBW as the case group and 140 neonates without LBW as the control group were selected from all male and female infants with the age of one year during the research through random sampling and using $\mathrm{Z}$ sample volume formula considering 0.05 alpha and test power of $80 \%$. Inclusion criteria were the chronological age of 12 months at the time of completing the questionnaire and birthplace of Yazd.

Exclusion criteria were being diagnosed with congenital abnormalities and non-Iranian nationality. At first, objectives of the research were explained to the parents and written informed consents were obtained prior to the study. In addition, the parents were ensured of the confidentiality terms regarding their personal information. Data collection tools were demographic and clinical characteristics questionnaire, and ages and stages questionnaire (ASQ). While the ASQ was filled by the parents, the demographic characteristics questionnaire was completed through interviews with mothers and evaluation of their medical files at the maternity hospital.

In this research, demographic characteristics questionnaire was designed considering the previous studies to gather background variables, including the type of delivery, type of nutrition, birth weight, maternal occupation, age and level of education, and Apgar at birth. As a global standard indicator for evaluation of the development of infants at different ages, the ASQ has a high reliability and validity and has been used in similar studies of the country with confirmed validity of $91 \%$, sensitivity of $90 \%$ and exclusiveness of $81-91 \%$ (11). This 19-item questionnaire is applied to 19 different age groups and is filled by parents. Each questionnaire contains 30 items of neonatal development, which are divided into five developmental areas, including communication, gross motor, fine motor, personal-social, and problemsolving. Each item of the questionnaire is allocated the score of 10 in case of the response of "yes", five for "often" and zero for the response of "not yet". In addition, the scores related to each developmental area are compared to the "cut-off point" announced in the sheet of "points of ASQ" for the same developmental domain in the desired age group.

The infants born before 37 weeks of the first day of the last menstrual period are known as premature newborns (12). Therefore, in the case of presence of a newborn with the history of premature birth, their corrected age would be estimated and the questionnaire would be filled at the corrected age of the neonates. After the extraction of information, data analysis was done by SPSS 19 (SPSS Inc., Chicago, Illinois, USA). The $t$-test was used to measure the developmental status between two groups; and Spearman's test was applied to study the correlation between the developmental status scores with quantitative variables; and P-value of less than 0.05 was considered significant.

\section{Results}

In this research, the case group had an average weight of $2171.4 \pm 339.8 \mathrm{gr}$, the mean age of mothers at delivery was $29.37 \pm 5.9$ years of which $93.6 \%$ were housewives, $50.7 \%$ were girls and $55 \%$ were breastfeeding. The control group had an average weight of $3222.5 \pm 390.8 \mathrm{gr}$, the mean age of mothers at delivery was $29.95 \pm 5.2$ years of which $85.7 \%$ were housewives, $52.1 \%$ were boys and $71.4 \%$ were breastfeeding. From the 280 neonates, 142 
$(50.7 \%)$ were male and $138(49.3 \%)$ were female with mean weight of $2696.9 \pm 640.9$ gr. The minimum and maximum weights at delivery were $700 \mathrm{gr}$ and $4470 \mathrm{gr}$, respectively. The highest frequency of normal delivery was observed in NBW neonates, whereas the highest frequency of cesarean was related to the LBW group. Moreover, the highest frequency of breastfeeding was found in the NBW group, and the highest frequency of feeding with formula was observed in the LBW group. There were 2 children with weights of less than $1000 \mathrm{gr}$,
7 children with 1000 to 1500 gr and 271 children more than 1500 gr. There was no significant difference between mean scores of developmental status in these children.

In total, the neonates had normal development in communication (99.3\%), gross motor (95.4\%), fine motor (98.6\%), problem-solving $(97.9 \%)$ and personal-social $(98.6 \%)$ areas. On the other hand, the highest frequency of developmental delay was allocated to the areas of gross motor and problem-solving (Figure 1).

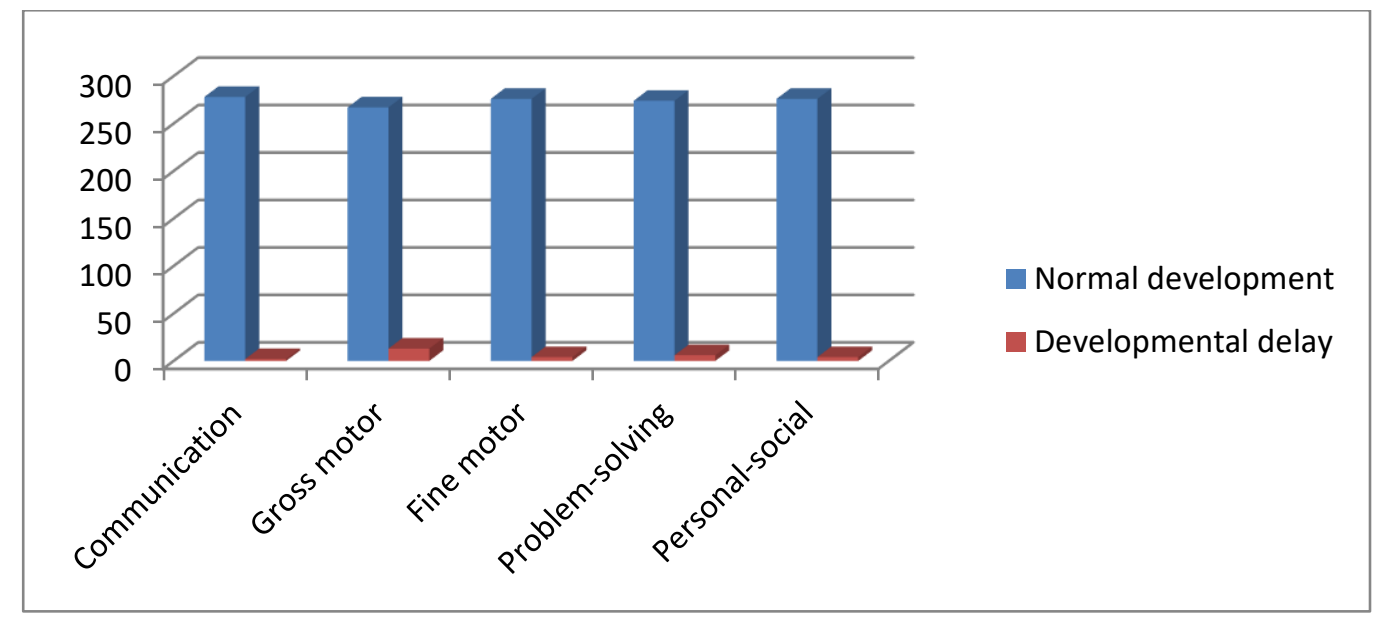

Figure 1. Frequency distribution of developmental delay in different separated fields.

In this study, the lowest mean score was related to the gross motor, whereas the highest mean was allocated to the fine motor skills. In the majority of areas, mean developmental score was lower in the LBW group, compared to the NBW group. The mean scores of personal-social were $56.04 \pm 8.15$ and $57.99 \pm 6.05$ in case group and control group, respectively. This difference was significant in the gross skill and personal-social areas $(P<0.005)($ Table 1$)$.

\section{Table 1. Comparison of mean scores of developmental areas in the evaluated groups}

\begin{tabular}{|c|c|c|c|}
\hline Developmental areas & $\begin{array}{c}\text { Case group } \\
\text { Mean (standard deviation) }\end{array}$ & $\begin{array}{c}\text { Control group } \\
\text { Mean (standard deviation) }\end{array}$ & Level of significance \\
\hline Communication & $56.05(6.57)$ & $56.80(6.50)$ & 0.301 \\
\hline Gross motor & $52.98(10.3)$ & $55.53(9.42)$ & 0.011 \\
\hline Fine motor & $58.60(3.98)$ & $58.00(6.53)$ & 0.888 \\
\hline Problem solving & $57.24(6.23)$ & $58.20(5.40)$ & 0.123 \\
\hline Personal-social & $56.04(8.15)$ & $57.99(6.05)$ & 0.005 \\
\hline
\end{tabular}

In this research, multiple regression model was employed to find factors affecting the development of neonates and eliminate the confounding effect of other variables. The results of each area are presented in Tables $2 \& \underline{3}$. According to these tables, we found significant correlation between gross motor, fine motor, problem solving and personal-social field with height at birth, birth rate respectively $(P<0.05)$ (Tables $\underline{2} \& \underline{3})$.
We found significant and positive correlation between weight, height and head circumference at birth and gross motor and personal-social areas $(P<0.05)$. Moreover, a reverse and significant association was found between time of hospitalization in NICU and gross motor area $(P<0.05)$ (Table 4). 
Table 2. Analysis of multiple regression related to the communication area, gross motor area and fine motor area

\begin{tabular}{|c|c|c|c|c|}
\hline \multirow{5}{*}{ 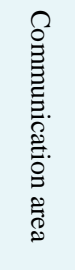 } & Variable & Beta & Standard error & Level of significance \\
\hline & Height at birth & 0.205 & 0.103 & 0.048 \\
\hline & Birth rate & -1.004 & 0.443 & 0.024 \\
\hline & Decreased blood sugar & -8.217 & 3.695 & 0.027 \\
\hline & Weak sucking & -15.783 & 6.427 & 0.015 \\
\hline \multirow{4}{*}{  } & Group & -2.463 & 1.162 & 0.035 \\
\hline & Tachypnea & -9.704 & 3.972 & 0.015 \\
\hline & Fever and seizure & -32.238 & 9.651 & 0.001 \\
\hline & Type of delivery & -1.974 & 1.174 & 0.094 \\
\hline \multirow{3}{*}{ 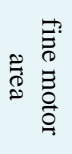 } & Birth rate & -0.737 & 0.350 & 0.036 \\
\hline & Fever and seizure & -29.495 & 5.126 & 0.000 \\
\hline & Gender & 1.030 & 0.611 & 0.093 \\
\hline
\end{tabular}

Table 3. Analysis of multiple regression related to problem-solving area and personal-social area

\begin{tabular}{|c|c|c|c|c|}
\hline \multirow{4}{*}{  } & Variable & Beta & Standard error & Level of significance \\
\hline & Time of hospitalization in NICU & -0.108 & 0.045 & 0.016 \\
\hline & Decreased blood sugar & -9.736 & 3.298 & 0.003 \\
\hline & Birth rate & -0.918 & 0.391 & 0.019 \\
\hline \multirow{3}{*}{ 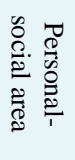 } & Height at birth & 0.542 & 0.111 & 0.000 \\
\hline & Birth rate & -1.230 & 0.479 & 0.011 \\
\hline & Decreased blood sugar & -8.587 & 3.998 & 0.033 \\
\hline
\end{tabular}

Table 4. Correlation between the score of different areas and some variables of the research

\begin{tabular}{|c|c|c|c|c|c|}
\hline \multirow[b]{2}{*}{ Variable } & \multicolumn{5}{|c|}{ Spearmsan's correlation coefficient } \\
\hline & $\begin{array}{l}\text { Communication } \\
\text { score }\end{array}$ & Gross motor score & Fine motor score & $\begin{array}{c}\text { Problem solving } \\
\text { score }\end{array}$ & Personal-social score \\
\hline Weight at birth & 0.074 & 0.166 & -0.010 & 0.060 & 0.149 \\
\hline P-value & 0.219 & 0.005 & 0.864 & 0.314 & 0.012 \\
\hline Head circumference & 0.043 & 0.145 & -0.028 & 0.054 & 0.191 \\
\hline P-value & 0.475 & 0.015 & 0.636 & 0.366 & 0.001 \\
\hline Height at birth & 0.085 & 0.171 & -0.021 & 0.061 & 0.197 \\
\hline P-value & 0.157 & 0.004 & 0.726 & 0.307 & 0.001 \\
\hline $\begin{array}{c}\text { Time of } \\
\text { hospitalization at } \\
\text { NICU }\end{array}$ & -0.011 & -0.119 & 0.035 & -0.074 & -0.006 \\
\hline P-value & 0.850 & 0.046 & 0.564 & 0.219 & 0.279 \\
\hline
\end{tabular}

\section{Discussion}

According to our findings, $6.07 \%$ (17 subjects) out of the 280 infants were not normal at least in one area and $93.57 \%$ (262 cases) were reported normal in all areas. The highest and the lowest levels of developmental delay were also associated with the gross motor skills and communication areas while the results of the descriptive cross-sectional research conducted by Dareh and Fatahi Bayat on 1444-to-60-month infants with a history of hospitalization in neonatal intensive care units (NICUs) in the city of Arak, Iran, indicated that $26.3 \%$ (30 cases) of the study subjects had abnormal development in a 
minimum of one area, and $6 \%$ of them had developmental delay in all areas. Besides, the highest and the lowest levels of developmental delay were related to the areas of communication and problem-solving, respectively (9).

Moreover, Boskabadi et al. carried out a cohort study on 270 premature newborns in the city of Mashhad, Iran, and evaluated infants' developmental status within the first two years of life. Based on the results of this research; $56 \%, 42.7 \%, 37.5 \%$, and $18 \%$ of the subjects aged 6,12 , 18 and 24 months, respectively had some degrees of developmental delay. The highest and the lowest levels of problems were also observed in problem-solving and communication areas in the given study, respectively (12). Consistently, in the current research, the lowest level of problems was found in the communication area. On the other hand, the findings of the cohort study by Tavasoli $e t$ $a l$., on 88 infants revealed that developmental delay had been observed in fine motor skills in neonates with LBW. However, no significant difference was found between the two study groups regarding gross motor skills in the mentioned study (2). In contrast, Akbari et al., shed light on the correlation between high-risk pregnancy and developmental delay in children aged 4-60 months and reported that the highest level of developmental delay was observed in the fine motor skills of the participants (8). The discrepancy between the results of these investigations and those of the present study was due to different age ranges of the subjects; i.e. children aged one year were evaluated in the present study.

According to the results of the present study, a significant relationship was also observed between LBW and gross motor and personal-social areas. In this regard, Islami et al., conducted a research entitled "Growth parameters of NICU admitted low birth weight preterm neonates at corrected ages of 6 and 12 month". Then followed up 50 premature and LBW infants admitted to the Shahid Sadoughi Hospital in the city of Yazd for one year, and consequently assessed their developmental status at the ages of 60 and 12 months using the ASQ. This research study indicated that problems in the developmental area (gross and fine motor skills and problem-solving) along with the social skills had been more common at the ages of 6 and 12 months in LBW neonates. In general, these infants had developmental delay in the gross motor and social areas (3). In addition, Ali Abadi et al., conducted a cross-sectional research on 32 infants at the age of 8-12 months (18 infants with LBW and 14 infants with NBW) and compared the mean scores of gross motor skills in two groups of neonates. They demonstrated a significant difference between the groups in terms of the gross motor skills (10). On the other hand, Grantham-McGregor et al., carried out a cohort study on
131 term infants with LBW and 131 term neonates with appropriate birth weight (AWB) in the North-East of Brazil. The results of this study revealed that the mean scores of mental and psychomotor development were lower in neonates with LBW at the age of six months $(P<0.0001)$, which had been intensified at the age of 12 months (13).

In the cohort study conducted by Datar et al., in the United States, the mental and the motor development of VLBW and MLBW infants were compared with normal infants during their first two years of life. In this respect, slight side effects were reported in the psychomotor development within the first two years of life in neonates with LBW. In other words, LBW could have major adverse effects on the mental, physical and growth development status of neonates at the ages of 9 months and 2 years $(P<0.01)$ (14). In similar studies by Pin et al., in Australia (15) and Wilson-Costello (16), it was similarly indicated that premature neonates had a higher level of motor developmental delay compared to the newborns with normal weight. Furthermore, Ali Abadi et al., reiterated that neonates with LBW could be extremely exposed to being diagnosed with motor developmental abnormalities (10).

In addition, in a prospective cohort study by Modi et al., in 2013, which was conducted on 37 infants with VLBW and 35 newborns with NBW in India, the developmental indicators of neonates with VLBW were reported significantly lower as compared to those in infants with NBW. In general, they asserted that newborns with VLBW had a lower neurological development indicator at the age of one year compared to the infants with NBW (17). Other studies also confirmed the correlation between LBW and developmental delay $(\mathbf{8 , 1 8 )}$. However, Kardjati et al., found no difference in the motor development of neonates with LBW and those with normal weights during the first six months of their life in Madura, Indonesia (19).

The given difference between the results was due to the assessment of neonates with LBW, who were exclusively breastfed during their first 6 months of life in the research by Kardjati et al. On the other hand, Noohjah et al., concluded no significant relationship between birth weight and motor developmental abnormality (20). The cited research was also conducted on infants aged one-tofive years old and such a discrepancy in children's age might be the cause of inconsistency of results. A study by Jimenez Martin et al., was carried out on 166 low-weight neonates in Spain. The developmental status of the subjects was evaluated during the first 3 years of their life. At the end, cerebral palsy (CP) was found in $50 \%$ of these children, whereas speech and mental development in these subjects was reported to be at a normal level (21). 
In the present research, a significant relationship was observed between the personal-social areas and the variables of birth height, birth rate, and decreased blood sugar levels. In a systematic review, Ritchie et al., reported that very premature neonates had insufficient social development, and similar problems were also observed during their entire childhood. In addition, it was demonstrated that the given infants were exposed to the neurodevelopmental delay. Furthermore, the predictive factors of the social developmental status of children were age of pregnancy, neonatal brain abnormalities, and socioeconomic conditions of family (22). In the present study, a significant relationship was found between various developmental areas and birth rate which was in line with the results obtained by Karami et al. (11). However, no significant relationship was observed between birth rate and developmental delay in the research by Afraz et al., examining the developmental status in 4-to-24-month infants of adolescent mothers in 2013 (23).

In another research by Dareh and Fatahi Bayat, no significant correlation was observed between various developmental areas and the variables of gender, duration of hospitalization, and birth weight (9). In this regard, the results of the given study were in conflict with the findings of the present research regarding the duration of hospitalization and birth weight, in a way that a significant relationship was observed between some developmental areas and the variables of birth weight and duration of hospitalization in the present study. This inconsistency might be due to different research types and methods.

In a cross-sectional study by Schonhaut et al., conducted on 1667 newborns in Chile, a reverse correlation was also observed between risks of developmental delay and gestational age at birth (24). Moreover, Ritchie et al., conducted a systematic review of the social development status of very premature infants and considered the gestational age as a predictive factor for the social developmental status in infants (22). Similarly, Bos et al., in a systematic review, showed that pre-term birth was a confounding factor for the fine motor disorders (25). In the study by Noohjah et al., investigating motor development in 800 children (1-to-5year-old), the age of pregnancy was significantly correlated with the motor developmental abnormality of the subjects (20). On the other hand, Kazerouni et al. observed no significant difference between the developmental status of premature neonates and their birth weight and age of pregnancy (6). The same results were also obtained in the present study demonstrating that no significant relationship was observed between gestational age and developmental delay.
In 2007, in a study by Zhang et al., on 210 premature newborns with a history of admission to NICUs at the age of 1 year in Shanghai, China; these premature infants were exposed to a high risk of neurodevelopmental delay and the risk factors for the developmental delay were reported to be low parental levels of education, multiple pregnancies, severe intracranial hemorrhage, as well as apnea. Therefore, early intervention could improve the neurodevelopmental status of premature neonates at high risk of developmental delay (26). In a cohort study by Grantham-McGregor et al., a significant relationship was similarly observed between mental developmental indicators of infants with LBW. However, the same correlation was not found in AWB newborns (13).

On the other hand, Afraz et al., found no relationship between maternal levels of education and developmental delay (23). Likewise, the same results were obtained in the present research. However, the difference between the present study results and the cited ones might be due to the fact that the highest levels of education in parents were observed in the group of newborns with NBW, whereas the lowest levels of education (high school diploma and below high school diploma) were found in the group of neonates with weights below 2500 gr. Another factor affecting this inconsistency between the results might be the age of pregnancy. In the research reported by Karami et al., no significant correlation was observed between types of feeding and emergence of developmental delay (11) which was in line with the findings in the present study. In general, LBW was recognized as one of the factors affecting the development of neonates. However, the difference in the areas of history of low weight and prematurity in various studies might be due to different research designs and methods, age of pregnancy, as well as measures taken in the rehabilitation process of these infants.

\section{Conclusion}

The present study investigated the developmental status of 1-year-old children with a history of low birth weights (LBW) and normal birth weight (NBW). Researchers sought to recognize and reduce limitations to the possible extent to provide valid and generalizable results. Therefore, it is better to consider multiple pregnancy, mothers' body mass index (BMI), parents' kinship (familial marriage), household social status, and the abortion history in the future studies. The inadequacy of some hospital records was a limitation of this study leading to the impossibility of determining relationship of the premature rupture of membranes (PROM) and its duration with the evolutionary disorder. Therefore, it is suggested to investigate this relationship in future studies.

However, it is recommended that more attention to be paid to the reduction of premature births and LBW by the 
health policymakers in the future. In the clinical trial by Koldewijn et al., it was concluded that performing interventional programs to evaluate the neonates and the role of parents in the improvement of the conditions and creation of better neonatal status had a significant effect on the developmental status of newborns with LBW (27).

The present research also suggests increasing the quality of prenatal care to prevent the premature birth, performing developmental screening and follow-up of all newborns especially in LBW newborns, paying more attention to the developmental status of older children, and paying more attention to the hospital personnel in the neonatal intensive care unit (NICU), the blood sugar reduction, respiratory distress and seizures in newborns.

As a result, accurate and timely screening and informing parents through health promotion or the media plans can prevent the emergence of developmental disorders and the associated complications.

\section{Acknowledgements}

Hereby, we extend our gratitude to the Health Deputy of Shahid Sadoughi University of Medical Sciences, Mr. Mohammad Hassan Lotfi, for his contributions. In addition, we would like to thank all of the managers and personnel of comprehensive healthcare centers, Hospitals of Shahid Sadoughi, Madar, Shohadaye Kargar, Goodarz, Seyyed Al Shohada, Mortaz and Afshar, organization of endowment and charity and all mothers for their cooperation with the research.

\section{Conflict of Interest}

Authors declared no conflict of interests.

\section{References}

1. Soleimani F, Zaheri F, Abdi F. Developmental outcome of low birth-weight and preterm newborns: a re-view of current evidence. Tehran Univ Med Sci J. 2013; 71(9): 551-61.

2. Tavasoli A, Aliabadi F, Eftekhari R. Motor developmental status of moderately low birth weight preterm infants. Iran $\mathrm{J}$ Pediatr. 2014; 24(5): 581-6.

3. Islami Z, Fallah R, Mosavian T, Pahlavanzadeh MR. Growth parameters of NICU admitted low birth weight preterm neonates at corrected ages of 6 and 12 month. Iran J Reprod Med. 2012; 10(5): 459-64.

4. Zarei M, Paydar Z, Irandoost A. Maternal risk factors contributing to preterm birth. J North Khorasan Univ Med Sci. 2016; 7(4): 793-9. [DOI:10.29252/jnkums.7.4.793]

5. Abdeyazdan Z, Ehsanpour S, Hemmati E. Developmental millstones in childern with normal, loe and very low birth wights. Urmia Nurs Midwif Univ Med Sci J. 2013; 11(8): 570-7.

6. Kazerooni S, Keshavarz k, Abasi R, et al. Status of development of premature children from 4 to 12 months in the Neonatal Intensive Care Unit (NICU) Admission Based on the ASQ Questionnaire. Armaghane Danesh. 2014; 19: 780-7

7. Leadford AE, Warren JB, Manasyan A, et al. Plastic bags for prevention of hypothermia in preterm and low birth weight infants. Pediatrics. 2013; 132(1): e128-34 [DOI:10.1542/peds.2012-2030] [PMID] [PMCID]

8. Amiraliakbari S, Torabi F, Soleimani F, Alavi majd H. Correlation between high risk pregnancy and developmental delay In children 4-60 months in Isfahan 2010-2011. J Rehabil 2010; 11: 40-9. doi:10.3402/ljm.v7i0.18811 [DOI:10.3402/ljm.v7i0.18811] [PMID] [PMCID]

9. Dorre F, Fattahi bayat G. Evaluation of children's development (4-60) with history of NICU admission based on ASQ in Amir kabir Hospital, Arak. Ardabil Univ Med Sci J. 2011; 11(2): 143-50.

10. Aliabadi F, Nazi S, Maghfori B. Gross motor development of low birth weight infants with the history of being in Aliasghar hospital corrected aged 8 to 12 months. Modern Rehabil. 2011; 5(2): $35-40$

11. Karami K, Abbasi L, Moridi F, Falah F, Bayat Z, Pourvakhshoori N. Evaluation criteria and factors associated with the development of one year old children in Khorramabad. Iran J Pediatr Nurs. 2015; 1(3): 57-64.

12. Boskabadi H, Bagheri F, Askari Hosseni Z. Developmental disorders in preterm neonates during the first two years of life using the ages and stages questionnaire. Babol Univ Med Sci J. 2016; 18(2): 7-13.

13. Grantham-McGregor SM, Lira PI, Ashworth A, Morris SS, Assuncao AM. The development of low birth weight term infants and the effects of the environment in northeast Brazil. J Pediatr. 1998; 132(4): 661-6. [DOI:10.1016/S00223476(98)70357-9]

14. Datar A, Jacknowitz A. Birth weight effects on children's mental, motor, and physical development: evidence from twins data. Maternal Child Health J. 2009; 13(6): 780-94. [DOI:10.1007/s10995-009-0461-6] [PMID] [PMCID]

15. Pin TW, Darrer T, Eldridge BEV, Galea MP. Motor development from 4 to 8 months corrected age in infants born at or less than 29 weeks' gestation. Develop Med Child Neurol. 2009; 51(9): 739-45. [DOI:10.1111/j.14698749.2009.03265.x] [PMID]

16. Wilson-Costello $\mathrm{D}$. Is there evidence that long-term outcomes have improved with intensive care? Seminar Fet Neonat Med. 2007; 12(5): 344-54. doi:10.1016/j.siny.2007.06.010 [DOI:10.1016/j.siny.2007.06.010] [PMID]

17. Modi M, Saluja S, Kler N, et al. Growth and neurodevelopmental outcome of VLBW infants at 1 year corrected age. Indian Pediatrics. 2013; 50(6): 573-7. [DOI:10.1007/s13312-013-0170-5] [PMID]

18. Karimi M, Fallah R, Dehghanpoor A, Mirzaei M. Developmental status of 5-year-old moderate low birth weight children. Brain Develop. 2011; 33(8): 651-5. [DOI:10.1016/j.braindev.2010.10.022] [PMID]

19. Kardjati S, Kusin JA, De With C, Renqvist UH. Low birth weight babies under village conditions: feeding pattern, growth and motor development. Paediatrica Indonesiana. 1991; 3(3-4): 84-98.

20. Nouhjah S, Mokhveli Khazaei F, Mahdavi zadeh N. Assessment of motor development of children attending health centers of dezful city using world health organization standard indexes. J Paramed Sci Rehabil. 2014; 3(1): 16-26.

21. Jimenez Martin AM, Servera Ginard C, Roca Jaume A, Frontera Juan G, Perez Rodriguez J. Developmental outcome of extremely low birth weight infants $(<1,000 \mathrm{~g})$ during the 
first three years of life. Anales de pediatria (Barcelona, Spain : 2003) 2008; 68(4): 320-8. [DOI:10.1157/13117701] [PMID]

22. Ritchie K, Bora S, Woodward LJ. Social development of children born very preterm: a systematic review. Develop Med Child Neurol. 2015; 57(10): 899-918. [DOI:10.1111/dmen.12783] [PMID]

23. Afraz S, Ahmadi M, Sajedi F, Akbarzadeh Baghban A. Development status of 4-24 months children born to teenage mothers referred to health care centers in yasuj, 2013. Armaghane Danesh. 2015; 20(3): 253-63.

24. Schonhaut L, Armijo I, Perez M. Gestational age and developmental risk in moderately and late preterm and early term infants. Pediatrics. 2015; 135(4): 835-41. [DOI:10.1542/peds.2014-1957] [PMID]
25. Bos AF, Van Braeckel KN, Hitzert MM, Tanis JC, Roze E. Development of fine motor skills in preterm infants. Develop Med Child Neurol. 2013; 55(4): 1-4. [DOI:10.1111/dmen.12297] [PMID]

26. Zhang GQ, Shao XM, Lu CM, ed al. Neurodevelopmental outcome of preterm infants discharged from NICU at 1 year of age and the effects of intervention compliance on neurodevelopmental outcome. Chinese J Contem Pediatr. 2007; 9(3): 193-7.

27. Koldewijn K, Wolf MJ, van Wassenaer A, et al. The infant behavioral assessment and intervention program for very low birth weight infants at 6 months corrected age. J Pediatr. 2009; 154: 33-8. [DOI:10.1016/j.jpeds.2008.07.039] [PMID]

\section{How to Cite This Article:}

Dehghani A, Sobhani M, Nouri Shadkam M, Falahzadeh H, Mohammadi M, Sharifi A. Developmental Assessment in Low Birth Weight Infants at One Year of Age using Ages and Stages Questionnaire. J Adv Med Biomed Res. 2019; 27 (121) :8-15

\section{Download citation:}

$\underline{\text { BibTeX }}|\underline{\text { RIS }}| \underline{\text { EndNote }}|\underline{\text { Medlars }}| \underline{\text { ProCite }}|\underline{\text { Reference Manager }}| \underline{\text { RefWorks }}$

\section{Send citation to:}

Mendeley 2 Zotero RefWorks $\underline{\text { RefWorks }}$ 